\title{
Light vector meson photoproduction in hadron-hadron and nucleus-nucleus collisions at energies available at the CERN Large Hadron Collider
}

\author{
G. Sampaio dos Santos and M. V. T. Machado \\ High Energy Physics Phenomenology Group, GFPAE IF-UFRGS Caixa Postal 15051, CEP 91501-970, Porto Alegre, RS, Brazil
}

(Received 17 July 2014; revised manuscript received 7 January 2015; published 5 February 2015)

\begin{abstract}
In this work we analyze the theoretical uncertainties on the predictions for the photoproduction of light vector mesons in coherent $p p, p A$, and $A A$ collisions at the CERN Large Hadron Collider energies using the color dipole approach. In particular, we present our predictions for the rapidity distribution for $\rho^{0}$ and $\phi$ photoproduction and perform an analysis on the uncertainties associated to the choice of vector meson wave function and the phenomenological models for the dipole cross section. Comparison is done with the recent ALICE analysis on coherent production of $\rho^{0}$ at $2.76 \mathrm{TeV}$ in $\mathrm{PbPb}$ collisions.
\end{abstract}

DOI: 10.1103/PhysRevC.91.025203

PACS number(s): 12.38.Bx, 13.60.Hb, 25.10.+s, 25.75.Dw

\section{INTRODUCTION}

The exclusive photoproduction of vector mesons has been investigated recently both experimentally and theoretically [1-5]. In particular, the light vector mesons as $\rho$ and $\phi$ do not have a perturbative scale associated to the process in the photoproduction limit and so they test the nonperturbative regime of QCD. The transition between the perturbative hard treatment and the soft regime can be addressed by the so-called saturation approaches [7] within the color dipole formalism [6]. In those formalisms a saturation scale characterizes the limitation on the maximum phase-space parton density that can be reached in the hadron wave function. In such a framework, the typical scale driven by the dynamics of light meson production is the saturation scale and the photons can be considered as color dipoles in the mixed light cone representation, where their transverse size can be considered frozen during the interaction [8]. The corresponding scattering process is characterized by the color dipole cross section describing the interaction of those color dipoles with the nucleon or nucleus targets. Accordingly, the $q \bar{q}$ fluctuations, i.e., the color dipoles, of the incoming quasireal photon interacts with the target via the dipole cross section and the result is projected in the wave function of the observed hadron. At high energies, i.e., small- $x$ region, it is expected that a transition between the regime described by the linear dynamics of emissions chain and a new regime where the physical process of recombination of partons turns out to be crucial. The transition is driven by the saturation scale $Q_{\text {sat }} \propto A^{1 / 3} x^{\lambda}$, which is typically enhanced in the scattering on nuclei targets [7].

The approach shortly described above has done a good job in describing the vector meson photo and electroproduction at the DESY-HERA energy regime considering a proton target (see, e.g., Ref. [9]). The possibility for an investigation of the photonuclear production in similar energies was provided by the BNL Relativistic Heavy Ion Collider (RHIC) measurements on $\rho$ and $J / \Psi$ production considering the coherent gold-gold heavy ion collisions [10,11]. For a long time the analysis of coherent collisions in hadronic interactions as an alternative way to investigate the QCD dynamics at high energies has been proposed [12-21]. The basic idea in coherent hadronic collisions is that the cross section for a given process can be factorized in terms of the equivalent flux of photons into the hadron projectile and the photon-photon or photon-target production cross section [22]. The main advantage of using colliding hadrons and nuclear beams for studying photon-induced interactions is the high equivalent photon energies and luminosities achieved at RHIC and CERN Large Hadron Collider (LHC). Consequently, studies of $\gamma p$ or $\gamma A$ interactions at the LHC could provide valuable information on the QCD dynamics at high energies. The main point here is to investigate the robustness of the phenomenological models including the saturation phenomenon which have their parameters fixed by DESY-HERA data when extrapolated to the very high energy regime reached at the LHC.

Our goal in this work is to analyze the theoretical uncertainties on the predictions for the photoproduction of light vector mesons in coherent $p p, \mathrm{PbPb}$, and $p \mathrm{~Pb}$ collisions at the LHC using the color dipole approach. Predictions for the rapidity distribution for $\rho^{0}$ and $\phi$ photoproduction will be furnished and an analysis on the uncertainties associated to the choice of vector meson wave function and the phenomenological models for the dipole cross section is performed. Moreover, we will present our predictions for the rapidity dependence of $\rho$ cross sections at LHC energy of $2.76 \mathrm{TeV}$ in coherent $\mathrm{PbPb}$ collisions, which is currently under analysis by the ALICE collaboration [23].

\section{THEORETICAL FRAMEWORK}

The exclusive meson photoproduction in hadron-hadron collisions can be factorized in terms of the equivalent flux of photons of the hadron projectile and photon-target production cross section [22]. The photon energy spectrum, $d N_{\gamma}^{p} / d \omega$, which depends on the photon energy $\omega$, is well known [22]. The rapidity distribution $y$ for vector meson photoproduction in $p p$ collisions can be written as

$$
\begin{aligned}
\frac{d \sigma}{d y}(p p \rightarrow p \otimes V \otimes p)= & S_{\text {gap }}^{2}\left[\omega \frac{d N_{\gamma}^{p}}{d \omega} \sigma(\gamma p \rightarrow V p)\right. \\
& +(y \rightarrow-y)] .
\end{aligned}
$$


The produced state with mass $m_{V}$ has rapidity $y \simeq \ln \left(2 \omega / m_{V}\right)$ and the square of the $\gamma p$ center-of-mass energy is given by $W_{\gamma p}^{2} \simeq 2 \omega \sqrt{s}$. The absorptive corrections due to spectator interactions between the two hadrons are represented by the factor $S_{\text {gap }}$. For simplicity here, we did not consider absorption corrections. The photon-Pomeron interaction will be described within the light-cone dipole frame, where the probing projectile fluctuates into a quark-antiquark pair with transverse separation $r$ (and momentum fraction $z$ ) long after the interaction, which then scatters off the hadron. The cross section for exclusive photoproduction of vector meson off a nucleon target is given by [8]

$$
\begin{aligned}
\sigma(\gamma p \rightarrow V p) & =\frac{1}{16 \pi B_{V}}\left|\int d z d^{2} r \Phi_{T}^{\gamma^{*} V} \sigma_{\operatorname{dip}}\right|^{2}, \\
\Phi_{T}^{\gamma^{*} V} & =\sum_{h, \bar{h}} \Psi_{h, \bar{h}}^{\gamma}\left(z, r, m_{q}\right) \Psi_{h, \bar{h}}^{V *}\left(z, r, m_{q}\right),
\end{aligned}
$$

where $\Psi^{\gamma}\left(z, r, m_{q}\right)$ and $\Psi^{V}\left(z, r, m_{q}\right)$ are the light-cone wave function of the photon and of the vector meson, respectively. The Björken variable is denoted by $x=M_{V}^{2} /\left(W_{\gamma p}^{2}-m_{p}^{2}\right)$, the dipole cross section by $\sigma_{\text {dip }}(x, r)$, and the diffractive slope parameter by $B_{V}$. Here, we consider the energy dependence of the slope using the Regge motivated expression, $B_{V}\left(W_{\gamma p}\right)=$ $B_{0}+4 \alpha^{\prime} \log \left(W_{\gamma p} / W_{0}\right)$. We have considered $B_{0}=11 \mathrm{GeV}^{-2}$, $W_{0}=95 \mathrm{GeV}$, and $\alpha^{\prime}=0.25 \mathrm{GeV}^{-2}$ [24]. Similarly, the rapidity distribution $y$ in nucleus-nucleus collisions has the same factorized form,

$$
\begin{aligned}
\frac{d \sigma}{d y}(A A \rightarrow A \otimes V \otimes Y)= & {\left[\omega \frac{d N_{\gamma}^{A}}{d \omega} \sigma(\gamma A \rightarrow V+Y)\right.} \\
& +(y \rightarrow-y)],
\end{aligned}
$$

where the photon flux in the nucleus is denoted by $d N_{\gamma}^{A} / d \omega$ and $Y=A$ (coherent case) or $Y=A^{*}$ (incoherent case). The exclusive photoproduction off nuclei for coherent and incoherent processes can be simply computed in high energies where the large coherence length $l_{c} \gg R_{A}$ is fairly valid. The expressions for both cases are given by [25]

$$
\begin{aligned}
\sigma(\gamma A \rightarrow V A)= & \int d^{2} b\left|\left\langle\Psi^{V}\left|1-\exp \left[-\frac{1}{2} \sigma_{\mathrm{dip}} T_{A}\right]\right| \Psi^{\gamma}\right\rangle\right|^{2}, \\
\sigma\left(\gamma A \rightarrow V A^{*}\right)= & \frac{1}{16 \pi B_{V}} \int d^{2} b T_{A} \mid\left\langle\Psi^{V}\right| \sigma_{\mathrm{dip}}(x, r) \\
& \times\left.\exp \left[-\frac{1}{2} \sigma_{\mathrm{dip}} T_{A}(b)\right]\left|\Psi^{\gamma}\right\rangle\right|^{2},
\end{aligned}
$$

where $T_{A}(b)=\int d z \rho_{A}(b, z)$ is the nuclear thickness function. The notation $\left\langle\Psi^{V}|(\cdots)| \Psi^{\gamma}\right\rangle$ represents the overlap over the wave functions. The rapidity distribution for the case of coherent $p A$ collisions can be also obtained. Disregarding

\begin{tabular}{|c|c|c|c|c|c|c|c|}
\hline \multirow[t]{2}{*}{ V } & \multicolumn{3}{|c|}{$\begin{array}{l}\text { common } \\
\text { parameters }\end{array}$} & \multicolumn{2}{|c|}{$\begin{array}{c}\mathrm{BG} \\
\text { parameters }\end{array}$} & \multicolumn{2}{|c|}{$\begin{array}{c}\text { LCG } \\
\text { parameters }\end{array}$} \\
\hline & $M_{V}$ & $m_{f}$ & $\hat{e}_{f}$ & $R^{2}$ & $N_{T}$ & $R_{T}^{2}$ & $N_{T}$ \\
\hline$\rho$ & 0.776 & $0.14(0.01)$ & $1 / \sqrt{2}$ & 12.3 & 0.0259 & 21.0 & 4.47 \\
\hline$\phi$ & 1.019 & $0.14(0.01)$ & $1 / 3$ & 10.0 & 0.0251 & 16.0 & 4.75 \\
\hline
\end{tabular}
the contribution from photonuclear interaction, the simplified expression is given by

$$
\frac{d \sigma}{d y}(p A \rightarrow p \otimes V \otimes A)=\omega \frac{d N_{\gamma}^{A}}{d \omega} \sigma(\gamma A \rightarrow V A) .
$$

TABLE I. Parameters for the vector-meson light-cone wave functions [30,31] in units of $\mathrm{GeV}$.

In the numerical evaluations in next section, we have considered the boosted Gaussian [28] (BG) and the light-cone Gaussian [29] (LCG) wave functions and the phenomenological saturation models proposed in Refs. [26] (IIM model) and [27] [Golec-Biernat Wusthoff (GBW) model] which encode the main properties of the saturation approaches. The expressions for the overlap functions we have used appropriately summed over the helicity and flavor indices are given by

$$
\begin{aligned}
\Phi_{T}^{\gamma^{*} V}\left(z, r, m_{q}\right)= & \hat{e}_{f} \frac{\sqrt{4 \pi \alpha_{e}}}{(2 \pi)^{2}} N_{c}\left\{m_{f}^{2} K_{0}\left(r \epsilon_{f}\right) \phi_{T}(r, z)\right. \\
& \left.-\left[z^{2}+(1-z)^{2}\right] \epsilon_{f} K_{1}\left(r \epsilon_{f}\right) \partial_{r} \phi_{T}(r, z)\right\},
\end{aligned}
$$

where the constant $\hat{e}_{f}$ stands for an effective charge. It is given in Table I along with the quark and meson masses used. Here, $m_{f}$ denotes the mass of the quark with flavor $f$ and with $\epsilon_{f}^{2}=m_{f}^{2}$. For the BG wave functions [28], the function $\phi_{T}$ is given by

$$
\begin{aligned}
\phi_{T}= & N_{T} 4 \sqrt{2 \pi R^{2}} \exp \left[-\frac{m_{f}^{2} R^{2}}{8 z(1-z)}+\frac{m_{f}^{2} R^{2}}{2}\right. \\
& \left.-\frac{2 z(1-z) r^{2}}{R^{2}}\right] .
\end{aligned}
$$

The parameters $R$ and $N_{T}$ are constrained by unitarity of the wave funtion as well as by the electronic decay widths. They are given in Table I [30]. On the other hand, for the LCG wave function [29] one has the following expression:

$$
\phi_{T}=N_{T} z(1-z) \exp \left[-r^{2} /\left(2 R_{T}^{2}\right)\right]
$$

with the parameters also given in Table I [31]. The parameters for the meson wave functions shown in the table correspond to a fixed quark mass of $m_{q}=0.14 \mathrm{GeV}$. We have verified that there is some sensitivity when considering a smaller quark mass, which leads to a change in the overall normalization $N_{T}$ (it increases as $m_{q}$ diminishes).

The two wave functions considered above are samples of the available phenomenological models. For instance, for $\rho$ production, Forshaw and Sandapen [32-34] have used stateof-the-art constraints for the parameters of the meson wave function from recent data on $\rho$ electroproduction. In Ref. [32] the $\rho$ wave function was extracted from HERA data and it was found that it prefers a transverse wave function with enhanced end-point contributions. In Ref. [33] the leading twist-2 and subleading twist-3 distribution amplitudes of the $\rho$ meson have been extracted and in Ref. [34] they provided an antide Sitter/conformal field theory correspondence (AdS/CFT) holographic wave function for the $\rho$ meson and compare it to the available data on the photon-proton process. 
For the phenomenological models for the dipole-proton cross section, we have considered two sets of parameters for the IIM parametrization [26] (including charm quark in fits). In this case, the dipole cross section is parametrized as follows:

$$
\sigma_{\text {dip }}(x, r)=\sigma_{0} \begin{cases}0.7\left(\frac{\bar{\tau}^{2}}{4}\right)^{\gamma_{\text {eff }}(x, r)}, & \text { for } \bar{\tau} \leqslant 2, \\ 1-\exp \left[-a \ln ^{2}(b \bar{\tau})\right], & \text { for } \bar{\tau}>2,\end{cases}
$$

where $\bar{\tau}=r Q_{\text {sat }}(x)$ and the expression for $\bar{\tau}>2$ (saturation region) has the correct functional form, as obtained from the theory of the color glass condensate (CGC) [7]. For the color transparency region near saturation border $(\bar{\tau} \leqslant 2)$, the behavior is driven by the effective anomalous dimension $\gamma_{\text {eff }}(x, r)=$ $\gamma_{\mathrm{sat}}+\frac{\ln (2 / \tilde{\tau})}{\kappa \lambda y}$ with $\kappa=9.9$. The saturation scale is defined as $Q_{\text {sat }}^{2}(x)=\left(\frac{x_{0}}{x}\right)^{\lambda}$ and $\sigma_{0}=2 \pi R_{p}^{2}$.

The first set (labeled by IIM-old [35]) considers the previous DESY-HERA data and the values for parameters are $\gamma_{\mathrm{sat}}=0.7376, \lambda=0.2197, x_{0}=0.1632 \times 10^{-4}$, and $R_{p}=$ $3.344 \mathrm{GeV}^{-1}\left(\sigma_{0}=27.33 \mathrm{mb}\right)$. For IIM-old, the light quark mass is fixed as $m_{u, d, s}=0.14 \mathrm{GeV}$. The second set (labeled IIM-new [36]) considered the extremely small error bars on the recent ZEUS and $\mathrm{H} 1$ combined results for inclusive Deep Inelastic Scattering (DIS). In this case, the parameters are $\gamma_{\text {sat }}=0.762, \lambda=0.2319, x_{0}=0.6266 \times 10^{-4}$, and $\sigma_{0}=$ $21.85 \mathrm{mb}$. For IIM-new, the light quark mass is fixed as $m_{u, d, s}=10^{-2}-10^{-4} \mathrm{GeV}$ (we take $m_{q}=0.01$ in the numerical calculations). At the same $x$ value, the saturation scale is higher for IIM-new as $x_{0}$ and $\lambda$ are both bigger as for the IIM-old case. On the other hand, the asymptotic value of dipole cross section $\sigma_{0}$ is smaller for IIM-new. We also see that a smaller $\Phi_{T}^{\gamma^{*} V}\left(z, r, m_{q}\right)$ is obtained, which is directly dependent on $m_{q}$, when compared to the value of mass $m_{q}=0.14 \mathrm{GeV}$.

In order to compare the dependence on distinct models, we also consider the simple GBW parametrization [27], where the dipole cross section is given by

$$
\sigma_{\mathrm{dip}}(x, r)=\sigma_{0}\left[1-\exp \left(-\frac{r^{2} Q_{\mathrm{sat}}^{2}}{4}\right)^{\gamma_{\mathrm{eff}}}\right]
$$

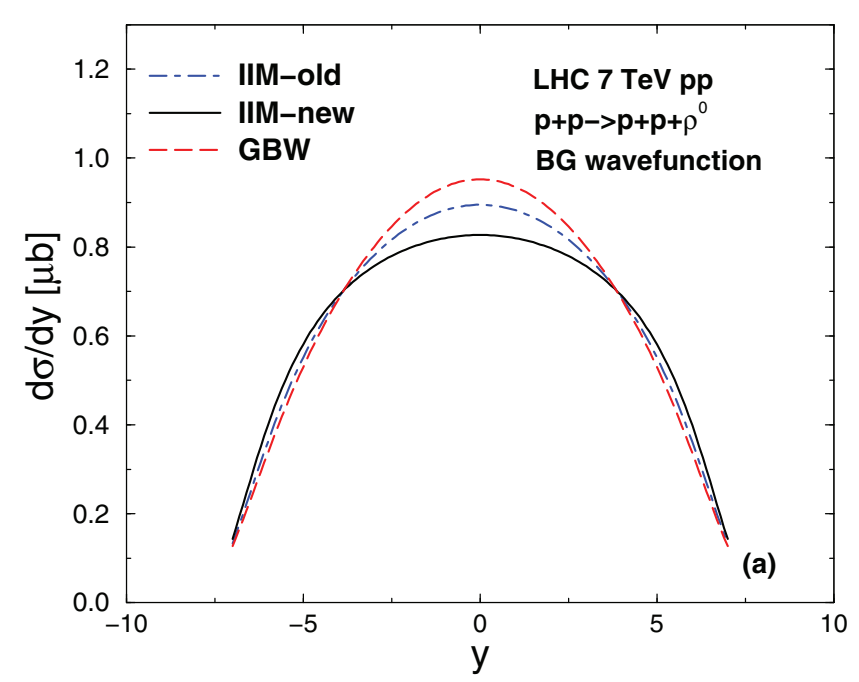

where the the effective anomalous dimension is taken as $\gamma_{\text {eff }}=1$.

As a final note on the details of the present calculation, we discuss the threshold correction, the real part of amplitude, and skewness effects. In all numerical calculations, we multiply the dipole cross sections above by a threshold correction factor $(1-x)^{n}$, where $n=5$ for light mesons and $n=7$ for the heavy ones (the value for $n$ is estimated using quark counting rules). The cross section in Eq. (2) has been computed including the real part of amplitude contribution and skewness correction in the following way:

$$
\hat{\sigma}_{\gamma p \rightarrow V p}=R_{g}^{2} \sigma(\gamma p \rightarrow V p)\left(1+\beta^{2}\right)
$$

with

$$
\begin{aligned}
& \beta=\tan \left(\frac{\pi \varepsilon}{2}\right), \quad R_{g}(\varepsilon)=\frac{2^{2 \varepsilon+3}}{\sqrt{\pi}} \frac{\Gamma(\varepsilon+5 / 2)}{\Gamma(\varepsilon+4)}, \\
& \varepsilon \equiv \frac{\partial \ln \left(\mathcal{A}^{\gamma p \rightarrow V p}\right)}{\partial \ln (1 / x)},
\end{aligned}
$$

where the factor $\left(1+\beta^{2}\right)$ takes into account the missing real part of the amplitude, with $\beta$ being the ratio of real to imaginary parts of the scattering amplitude. The factor $R_{g}$ incorporates the skewness effect, coming from the fact that the gluons attached to the $q \bar{q}$ can carry different light-front fractions $x, x^{\prime}$ of the proton. The skewness factor given in Eq. (10) was obtained at the NLO level, in the limit that $x^{\prime} \ll x \ll 1$ and at small $t$ assuming that the diagonal gluon density of target has a power-law form [37].

\section{RESULTS AND DISCUSSIONS}

Let us start by calculating the rapidity distribution for $\rho$ and $\phi$ production in proton-proton collisions at the energy of $7 \mathrm{TeV}$. In Fig. 1(a) the results for $\rho^{0}$ taking into account the BG wave function and some samples of phenomenological models for the dipole cross section are presented. The dotdashed curve stands for the IIM dipole cross section using previous values of its fitted parameters (IIM-old). The solid

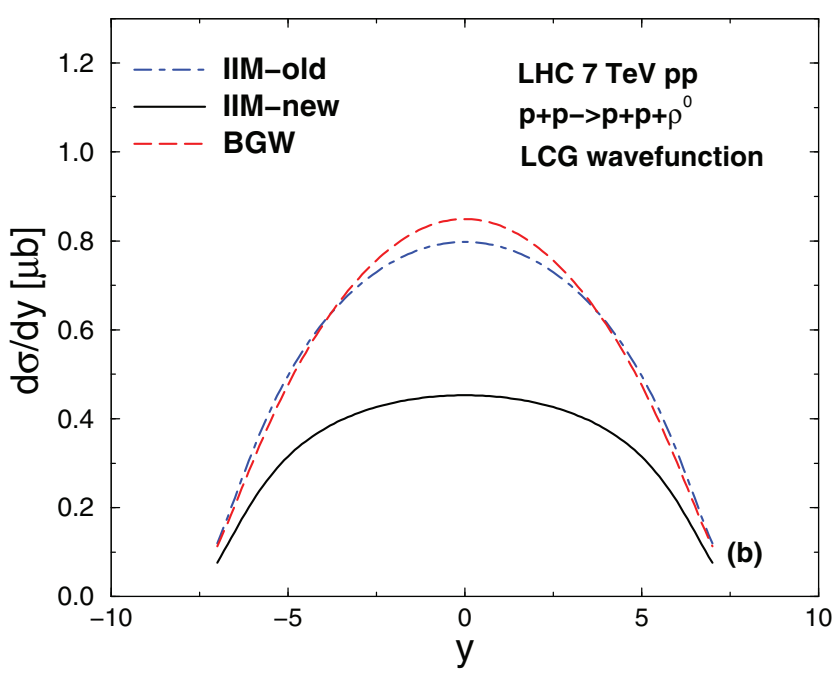

FIG. 1. (Color online) Predictions for the rapidity distribution of $\rho^{0}$ photoproduction in $p p$ collisions at $\mathrm{LHC}(\sqrt{s}=7 \mathrm{TeV})$ for the case of (a) boosted Gaussian (BG) and (b) light-cone Gaussian (LCG) wave functions and several models for the dipole cross section (see text). 

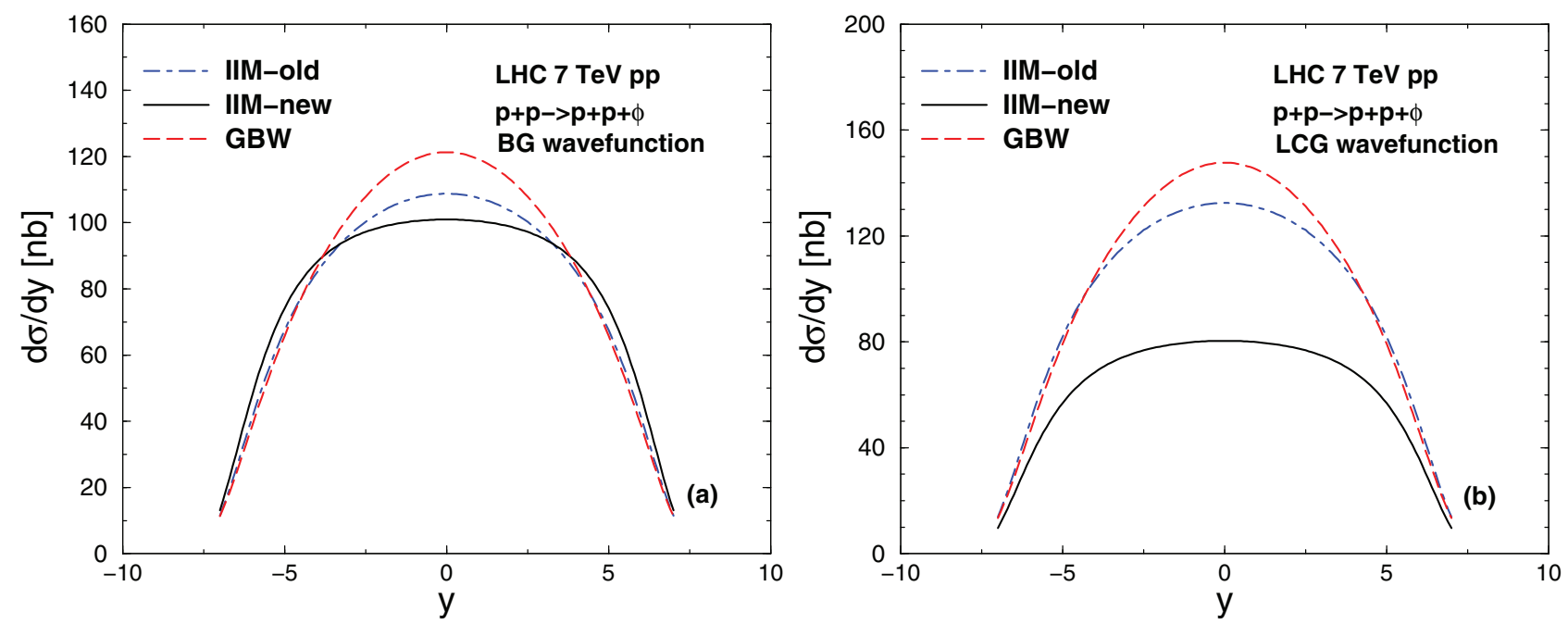

FIG. 2. (Color online) Predictions for the rapidity distribution of $\phi$ photoproduction in $p p$ collisions at $\mathrm{LHC}(\sqrt{s}=7 \mathrm{TeV})$ for the case of (a) BG and (b) LCG wave functions and several models for the dipole cross section (see text).

line represents the result using the new fitted parameters for the IIM model (IIM-new), whereas the dashed curve stands for the celebrated GBW parametrization. The behavior at large rapidities is similar for the distinct models. However, at midrapidities there is an evident model dependence. It is found that $d \sigma / d y(y=0)=0.9,0.83,0.95 \mu \mathrm{b}$ for IIM-old, IIM-new, and GBW, respectively. The deviation is of order $14 \%$ in that case. In Fig. 1(b), the results are now presented for the LCG wave function and the notation is the same as for the previous plot. In the midrapidity region one gets $d \sigma / d y(y=$ $0)=0.80,0.45,0.85 \mu \mathrm{b}$ for IIM-old, IIM-new, and GBW, respectively. The predictions using the $\mathrm{LCG}$ wave function are smaller than the BG wave function case. In addition, there is an intense suppression when the IIM-new model is considered (a reduction by a factor of 1.8). Concerning the $\phi$ meson production, in Fig. 2(a) the results using the BG wave function are presented and in Fig. 2(b) are the corresponding values for LCG wave function. The notation is the same as the plots for the $\rho$ case. At midrapidity it is found that $d \sigma / d y(y=$ $0)=108.8 \mathrm{nb}$ (IIM-old), $101 \mathrm{nb}$ (IIM-new), and $121.3 \mathrm{nb}$ (GBW) using the BG wave function and $d \sigma / d y(y=0)=$ $132.4 \mathrm{nb}$ (IIM-old), $80.4 \mathrm{nb}$ (IIM-new), and $147.7 \mathrm{nb}$ (GBW) considering the LCG wave function. In contrast with the $\rho$ case, the midrapidity values of cross sections are higher using the LCG instead of BG wave function by a factor $20 \%$ at least for IIM-old and GBW models. This can be due to the richer structure of the $\mathrm{BG}$ wave function in comparison to the LCG wave function. Once again, a reduction is observed using the IIM-new model. As predictions for the $14 \mathrm{TeV}$ run, it is found that $d \sigma_{\rho} / d y(y=0)=0.71 \pm 0.21 \mu \mathrm{b}$ (IIM-new) and $d \sigma_{\rho} / d y(y=0)=1.04 \pm 0.06 \mu \mathrm{b}(\mathrm{GBW})$. The errors take into account the dependence on the wave function. For the $\phi$ case we get $d \sigma_{\phi} / d y(y=0)=99 \pm 11 \mathrm{nb}$ (IIMnew) and $d \sigma_{\phi} / d y(y=0)=153 \pm 16 \mathrm{nb}(\mathrm{GBW})$. The large theoretical uncertainty presented here it was also found when considering the pQCD $k_{\perp}$-factorization approach in [38,39], where the authors also considered the absorption effects. Our predictions are somewhat consistent with those in Refs. [38,39] at midrapidity for $14 \mathrm{TeV}$.

Now, we investigate the photonuclear production of $\rho$ and $\phi$ mesons in nucleus-nucleus collisions at the LHC. We will consider $\mathrm{PbPb}$ collisions at the energy of $2.76 \mathrm{TeV}$. In Fig. 3(a) we present the results for the rapidity distributions for the coherent $\rho$ production, $\mathrm{Pb}+\mathrm{Pb} \rightarrow \mathrm{Pb}+\rho^{0}+\mathrm{Pb}$, considering the $\mathrm{BG}$ wave function (without nuclear breakup). The preliminary ALICE data [23] for coherent $\rho$ production, $\frac{d \sigma}{d y}(y=0)=420 \pm 10$ (stat.) ${ }_{-55}^{+30}$ (syst.) $\mathrm{mb}$, is also presented and the notation for the curves is the same as for the protonproton case. It was found that $d \sigma / d y(y=0)=661.5 \mathrm{mb}$ (IIMold), $747 \mathrm{mb}$ (IIM-new), and $685.6 \mathrm{mb}$ (GBW), respectively. In any case, the predictions are in average $50 \%$ larger than the experimental result. The theoretical uncertainty associated to the model for the dipole cross section remains as in the proton-proton case. It is a distinction compared to the proton-proton case for the IIM-new for BG wave function, where the prediction is larger than IIM-old and GBW. A careful analysis on the quark mass dependence for the BG wave function would be in order. In Fig. 3(b) one presents the results considering the LCG wave function, including the previous BG prediction [40] (dotted line) that also considered the color dipole approach. This time we obtained the values $d \sigma / d y(y=0)=469.5 \mathrm{mb}(\mathrm{GM}), 585.1 \mathrm{mb}$ (IIM-old), $409 \mathrm{mb}$ (IIM-new), and $603.3 \mathrm{mb}(\mathrm{GBW})$, respectively. The results are smaller than those for the BG wave function and the IIM-new result is consistent with data within the error bars. In Fig. 4 we present the integrated cross section (all rapidities) as a function of $N N$ energy, including the lower energy $\left(\sqrt{s_{N N}}=\right.$ $62.4,130$, and $200 \mathrm{GeV}$ ) results from STAR Collaboration at RHIC [10]. The preliminary data from ALICE, $\sigma_{\text {total }}^{\text {coh }}=$ $4.3 \pm 0.1$ (stat.) ${ }_{-0.5}^{+0.6}$ (syst.) $\mathrm{b}$ is also presented. The predictions using the IIM-new (solid line) and GBW (dashed line) models for the dipole cross section and the LGC wave function are shown. When comparing the models investigated here to those data we have previously presented, the coherent cross section 

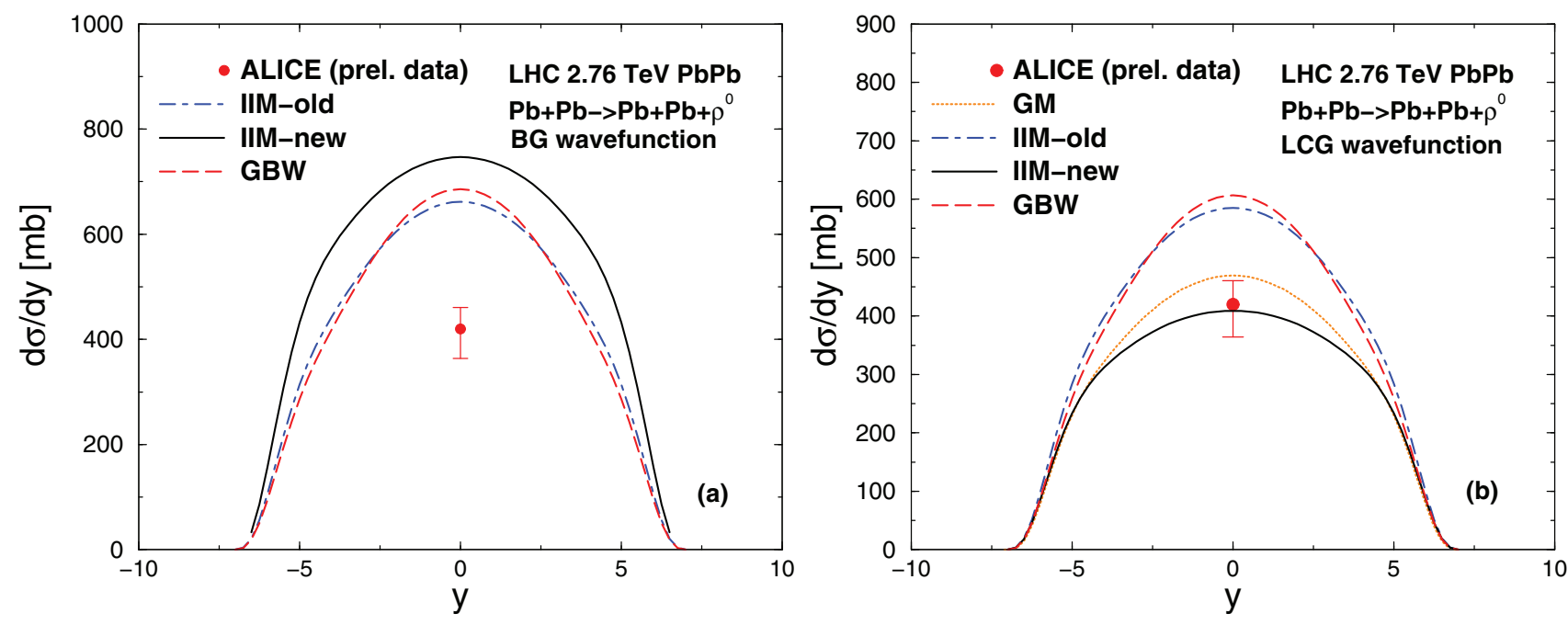

FIG. 3. (Color online) Predictions for the rapidity distribution of $\rho^{0}$ photonuclear production in $\mathrm{PbPb}$ collisions at $\mathrm{LHC}(\sqrt{s}=2.76 \mathrm{TeV})$ for the case of (a) BG and (b) LCG wave functions and several models for the dipole cross section (see text). The preliminary ALICE data [23] for central rapidity is also presented.

only (we did not include contributions with nuclear break up [41]) and a interpolation from RHIC to LHC are performed. It is verified that the color dipole approach gives a reasonable description of energy dependence from low to high energies. As a prediction for the future $5.5 \mathrm{TeV}$ run, we obtain the following the cross sections $\sigma_{0 n 0 n}^{\text {coh }}($ all $y)=5.30(7.21) \mathrm{b}$ using the LCG wave function and models IIM-new (GBW) for the dipole cross section. Our predictions are smaller (for this particular choice of wave function) than those presented in Ref. [42] Rebyakova, Strikman, and Zhalov (RSZ) where it is found that $\sigma_{0 n 0 n}^{\mathrm{RSZ}}($ all $y)=8.309 \mathrm{~b}$ at $5.5 \mathrm{TeV}$.

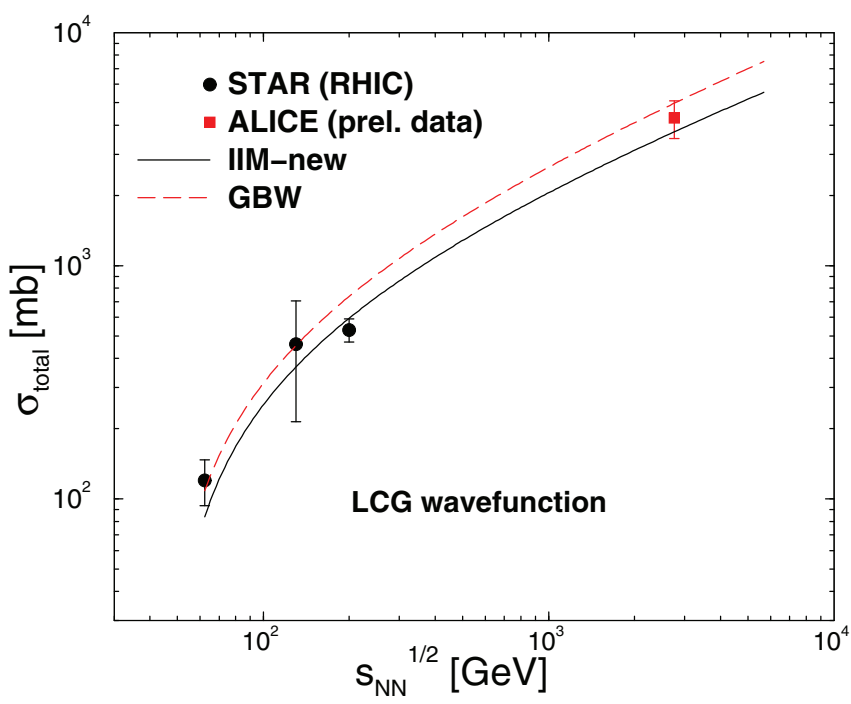

FIG. 4. (Color online) The total cross section for $\rho^{0}$ coherent production in nucleus-nucleus collisions as a function of energy $\sqrt{s_{N N}}$. The curves are for IIM-model (solid line) and GBW (dashed line) models for dipole cross section and both considering LCG wave function. The measurements of STAR (RHIC) [10] at low energy and the preliminary ALICE data [23] are also presented.
In Fig. 5 the results for the incoherent $\rho^{0}$ photonuclear production, $\mathrm{Pb}+\mathrm{Pb} \rightarrow \mathrm{Pb}+\rho^{0}+\mathrm{Pb}^{(*)}$, using same notation as previous plots are presented. In a similar way as for the coherent case, the predictions using the BG wave function (thick lines) are larger that the LCG option (thin lines) and can introduce an uncertainty by a factor of two (see IIM-new case) at midrapidities. The main prediction is that the incoherent $\rho$ production is of order $30 \pm 10 \mathrm{mb}$ at $y=0$ for energy of $2.76 \mathrm{TeV}$. Finally, the rapidity distribution for the $p A$ interaction is shown in Fig. 6, where the results for BG (thick lines) and LCG (thin lines) wave functions are presented in a single plot. We can see a strong dependence on the choice of meson wave function and on the dipole cross section,

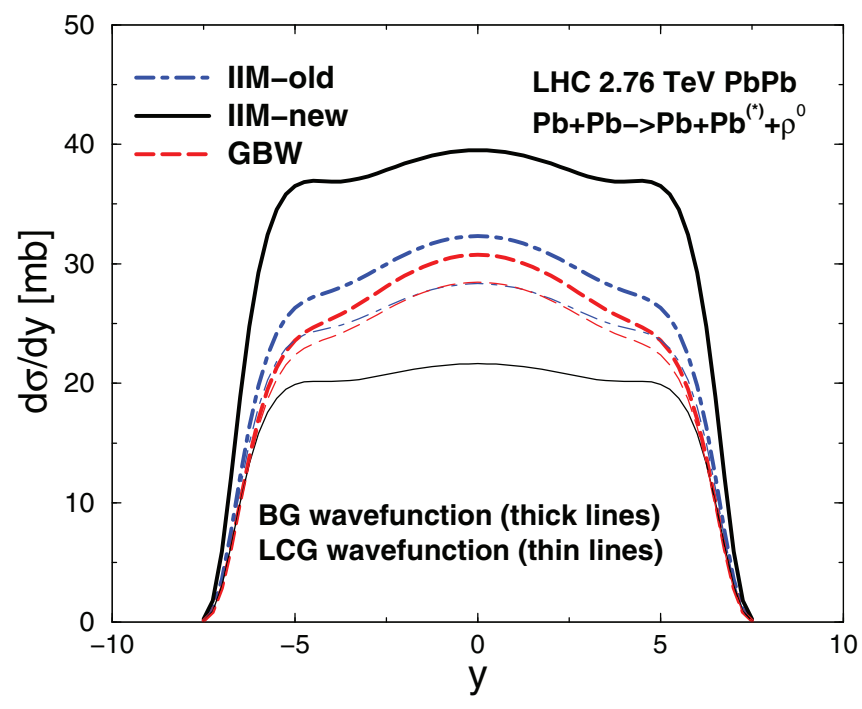

FIG. 5. (Color online) The rapidity distribution for $\rho^{0}$ incoherent production in $\mathrm{PbPb}$ collisions at $2.76 \mathrm{TeV}$. The thick lines represent the predictions using BG wave function and the thin lines represent the predictions for LCG wave function. 


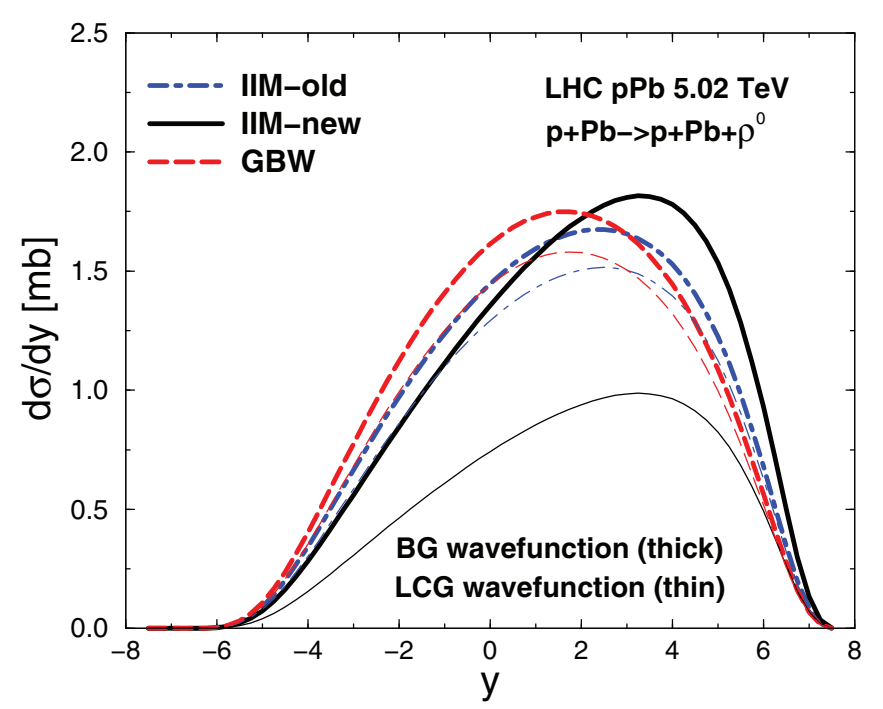

FIG. 6. (Color online) The rapidity distribution for $\rho^{0}$ coherent production in $p \mathrm{~Pb}$ collisions at $5.02 \mathrm{TeV}$. The thick lines represent the predictions using the BG wave function and the thin lines represent the predictions for the LCG wave function.

where the smaller cross section is provided by the IIM-new parametrization and LCG wave function. The pattern presented for the $\rho$ production is similar to the results for quarkonium production recently investigated in Refs. [43,44] also using the color dipole approach.

In summary, in this work we performed calculations considering the color dipole approach leading to predictions for the light vector meson production as $\rho$ and $\phi$ in coherent and incoherent interactions at the LHC energies for $p p, p \mathrm{~Pb}$, and $\mathrm{PbPb}$ collisions. We show that the theoretical uncertainty is considerably large and the main sources are the models for the meson wave function and the phenomenological models for the dipole cross section. The BG wave function leads to larger cross sections at midrapidity compared to the LCG wave function and the dependence of the overall normalization with the color dipole model is important. In particular, the recent ALICE preliminary data seem to favor the LCG wave function and more recent parametrizations for the dipole cross section. Our results demonstrate that the production rates in RHIC and LHC are fairly described by the color dipole approach. This corroborates the previous studies on heavy meson production as $J / \psi$ and $\psi(2 S)$ [45] (see also Fig. 7 from Ref. [2]) using the same framework presented here.

\section{ACKNOWLEDGMENTS}

This work was partially financed by the Brazilian funding agency Conselho Nacional de Desenvolvimento Científico e Tecnológico (CNPq) and by the French-Brazilian scientific cooperation project Coordenação de Aperfeiçoamento de Pessoal de Nível Superior (CAPES)-COFECUB 744/12. M.V.T.M. thanks Amir Rezaeian for useful discussions.
[1] R. Aaij et al. (LHCb Collaboration), J. Phys. G 40, 045001 (2013).

[2] R. Aaij et al. (LHCb Collaboration), J. Phys. G 41, 055002 (2014).

[3] B. Abelev et al. (ALICE Collaboration), Phys. Lett. B 718, 1273 (2013).

[4] E. Abbas et al. (ALICE Collaboration), Eur. Phys. J. C 73, 2617 (2013).

[5] B. Abelev et al. (ALICE Collaboration), Phys. Rev. Lett. 113, 232504 (2014).

[6] N. N. Nikolaev and B. G. Zakharov, Phys. Lett. B 332, 184 (1994); Z. Phys. C 64, 631 (1994).

[7] F. Gelis, E. Iancu, J. Jalilian-Marian, and R. Venugopalan, Ann. Rev. Nucl. Part. Sci. 60, 463 (2010); H. Weigert, Prog. Part. Nucl. Phys. 55, 461 (2005); J. Jalilian-Marian and Y. V. Kovchegov, ibid. 56, 104 (2006).

[8] J. Nemchik, N. N. Nikolaev, E. Predazzi, and B. G. Zakharov, Phys. Lett. B 374, 199 (1996).

[9] V. P. Goncalves, M. V. T. Machado, and A. R. Meneses, Eur. Phys. J. C 68, 133 (2010).

[10] G. Agakishiev et al. (STAR Collaboration), Phys. Rev. C 85, 014910 (2012).

[11] A. Afanasiev et al. (PHENIX Collaboration), Phys. Lett. B 679, 321 (2009).

[12] V. P. Goncalves and M. V. T. Machado, Eur. Phys. J. C 28, 71 (2003); 29, 37 (2003).

[13] V. P. Goncalves, M. V. T. Machado, and W. K. Sauter, Eur. Phys. J. C 46, 219 (2006).
[14] V. P. Goncalves and M. V. T. Machado, Eur. Phys. J. C 31, 371 (2003).

[15] V. P. Goncalves and M. V. T. Machado, Eur. Phys. J. C 40, 519 (2005).

[16] V. P. Goncalves and M. V. T. Machado, Phys. Rev. D 71, 014025 (2005).

[17] V. P. Goncalves and M. V. T. Machado, Phys. Rev. C 73, 044902 (2006).

[18] V. P. Goncalves and M. V. T. Machado, Phys. Rev. D 75, 031502 (2007).

[19] V. P. Goncalves and M. V. T. Machado, Phys. Rev. D 77, 014037 (2008).

[20] V. P. Goncalves and M. V. T. Machado, Phys. Rev. C 80, 054901 (2009).

[21] V. P. Goncalves, M. V. T. Machado, and A. R. Meneses, Phys. Rev. D 80, 034021 (2009).

[22] G. Baur, K. Hencken, D. Trautmann, S. Sadovsky, and Y. Kharlov, Phys. Rep. 364, 359 (2002); C. A. Bertulani, S. R. Klein, and J. Nystrand, Ann. Rev. Nucl. Part. Sci. 55, 271 (2005); K. Hencken et al., Phys. Rep. 458, 1 (2008).

[23] J. Nystrand (ALICE Collaboration), Nucl. Phys. A 931, 298 (2014).

[24] S. Aid et al. (H1 Collaboration), Nucl. Phys. B 463, 3 (1996).

[25] B. Z. Kopeliovich and B. G. Zakharov, Phys. Rev. D 44, 3466 (1991).

[26] E. Iancu, K. Itakura, and S. Munier, Phys. Lett. B 590, 199 (2004). 
[27] K. Golec-Biernat and M. Wüsthoff, Phys. Rev. D 59, 014017 (1998); 60, 114023 (1999).

[28] J. Nemchik, N. N. Nikolaev, E. Predazzi, and B. G. Zakharov, Z. Phys. C 75, 71 (1997).

[29] H. G. Dosch, T. Gousset, G. Kulzinger, and H. J. Pirner, Phys. Rev. D 55, 2602 (1997); G. Kulzinger, H. G. Dosch, and H. J. Pirner, Eur. Phys. J. C 7, 73 (1999).

[30] J. R. Forshaw, R. Sandapen, and G. Shaw, Phys. Rev. D 69, 094013 (2004).

[31] C. Marquet, R. Peschanski, and G. Soyez, Phys. Rev. D 76, 034011 (2007).

[32] J. R. Forshaw and R. Sandapen, J. High Energy Phys. 11 (2010) 037.

[33] J. R. Forshaw and R. Sandapen, J. High Energy Phys. 10 (2011) 093.

[34] J. R. Forshaw and R. Sandapen, Phys. Rev. Lett. 109, 081601 (2012).

[35] G. Soyez, Phys. Lett. B 655, 32 (2007).
[36] A. H. Rezaeian and I. Schmidt, Phys. Rev. D 88, 074016 (2013).

[37] A. G. Shuvaev, K. J. Golec-Biernat, A. D. Martin, and M. G. Ryskin, Phys. Rev. D 60, 014015 (1999).

[38] A. Cisek, W. Schäfer, and A. Szczurek, Phys. Lett. B 690, 168 (2010).

[39] A. Cisek, W. Schäfer, and A. Szczurek, Nucl. Phys. B, Proc. Suppl. 219-220, 56 (2011).

[40] V. P. Goncalves and M. V. T. Machado, Phys. Rev. C 84, 011902(R) (2011).

[41] A. J. Baltz, S. R. Klein, and J. Nystrand, Phys. Rev. Lett. 89, 012301 (2002).

[42] V. Rebyakova, M. Strikman, and M. Zhalov, Phys. Lett. B 710, 647 (2012).

[43] G. S. dos Santos and M. V. T. Machado, Phys. Rev. C 89, 025201 (2014).

[44] V. P. Gonçalves, B. D. Moreira, and F. S. Navarra, Phys. Rev. C 90, 015203 (2014).

[45] M. B. Gay Ducati, M. T. Griep, and M. V. T. Machado, Phys. Rev. D 88, 017504 (2013). 